\title{
LA RECONSTRUCCIÓN DE UN PASADO LINGÜÍSTICO A TRAVÉS \\ DE LA NARRATIVA PRESENTE: LENGUA, HISTORIA E IDENTIDAD \\ EN El NaCimiEnTo de los Negros
}

José ESTEBAN HERNANDEZ*

\section{Resumen}

El Nacimiento de los Negros, el asentamiento más importante de los negros mascogos, se encuentra dentro de los límites municipales de Músquiz, Coahuila, México. La lengua original y mayoritaria de la comunidad era hasta hace algunas décadas el afroseminol, una lengua emparentada al criollo de base inglesa que aún se habla en el sureste de los Estados Unidos. En el siguiente trabajo trazo la historia sociolingüística de la comunidad, basándome en las referencias históricas disponibles en la memoria colectiva y en la evidencia histórica con que cuenta la comunidad misma. Concilio dos fuentes que nos proporcionarán datos y pruebas que nos permitirán delinear la trayectoria lingüística del grupo. Utilizo los aspectos de la historia escrita que me son disponibles y que se centran en los principales acontecimientos que han experimentado los mascogos como grupo en el área fronteriza. Incorporo también el análisis de narrativas recogidas en entrevistas sociolingüísticas que se hicieron entre informantes de edad ya muy avanzada en la comunidad que asumen una identidad mascoga por ser descendientes directos de padre o madre de origen afroseminol. El análisis señala que el desplazamiento lingüístico, como resultado del contacto cultural en Nacimiento de los Negros, inició la integración de los negros mascogos a la cultura nacional. Sin embargo, el contexto sociohistórico marcó la pauta del proceso de aculturación. En el presente trabajo ofrezco datos testimoniales que apoyan las cuatro fases sociohistóricas, que arguyo, influyeron en dicho proceso: 1) la etapa de arrendamiento militar y persecución, 2) la etapa de transición y neutralidad, 3) la etapa de fuerte integración a la cultura nacional y 4) la etapa de integración y dependencia económica. Las etapas se distinguen entre sí por el mantenimiento del criollo de base inglesa en la primera fase, el inicio de la desplazamiento en las siguientes y el desplazamiento casi total en la última.

Palabras ClaVE: identidad, negros mascogos, mosaico lingüístico mexicano.

* Professor PhD da University of Texas - Pan American Languages and Literature. Edinburg, Texas.

E-mail: jhernandez52@utpa.edu 


\section{INTRODUCCIÓN}

Dentro del diverso y complejo mosaico lingüístico mexicano, resalta el caso del pueblo de Nacimiento de los Negros, Coahuila, por ser éste el único lugar en México en donde se habla gullah, un criollo de base inglesa (CBI de aquí en adelante), que ha estado en contacto hasta el día de hoy con el español de la región (LIPSKI, 2005). Sin embargo, a pesar de ser éste un caso excepcional en el contexto nacional mexicano, es poco lo que se sabe sobre las repercusiones que la situación de contacto ha tenido en la producción lingüística de los mascogos, habitantes de Nacimiento y descendientes de negros cimarrones que llegaron a México procedentes de los Estados Unidos a mediados del siglo diecinueve. ${ }^{1}$ Asimismo, se sabe aún menos sobre la historia lingüística de la localidad, que seguramente debió comprender claras etapas diferenciadas entre sí por el tipo de producción lingüística de los habitantes de Nacimiento durante los diferentes periodos históricos desde su llegada a México. Por ejemplo, es muy probable que durante los primeros años en suelo mexicano prevaleciera el predominio del gullah. Por lo tanto, es casi irrefutable que la mayoría de la comunidad se haya caracterizado en una primera instancia por un monolingüismo en CBI, y que el bilingüismo haya aparecido, en diferentes medidas para los distintos miembros de la comunidad, conforme se daba la integración de la comunidad a los patrones lingüísticos y culturales de la región y del ámbito nacional.

Los mascogos se establecieron en la Florida tras escapar de la esclavitud que se les imponía en las plantaciones inglesas y posteriormente estadounidenses en lo que es hoy el sureste norteamericano. En 1843, los mascogos, que para entonces habían sido forzados a establecerse en el estado norteamericano de Oklahoma, firmaron un acuerdo con el gobierno mexicano que les permitió asentarse en el estado mexicano de Coahuila a cambio de proteger la región fronteriza de las incursiones indígenas que la asolaban (RodRíguez, 1995, p. 101-102). Durante todo este tiempo, los mascogos han estado en contacto con otros pueblos de la región de Músquiz, Coahuila, predominantemente mestizos e hispanohablantes. En Brackettville, Texas donde se estableció otro grupo de cimarrones negros, más comúnmente conocidos como afroseminoles, los hablantes de CBI entraron en contacto tanto con mexicoamericanos 
(probablemente bilingües hispano y anglohablantes) como con angloamericanos (principalmente anglohablantes). Esta situación de contacto es única en México pues en ninguna otra parte del territorio se ha documentado el contacto a nivel social entre el español y un CBI.

El Nacimiento de los Negros, el asentamiento más importante de los negros mascogos, se encuentra dentro de los límites municipales de Músquiz, Coahuila. Su población consiste de unas 60 familias unidas por lazos de parentesco y que comparten un pasado común que los distingue de las comunidades vecinas. La lengua original y mayoritaria de la comunidad era hasta hace algunas décadas el afroseminol, una lengua emparentada al CBI que aún se habla en cierta medida en el área de las Carolinas y Georgia en el sureste de los Estados Unidos (CASIDY, 1986; JoNES-JACKSON, 1978, 1984, 1986, 1987). En cuanto a la descripción léxica, fonológica y sintáctica del afroseminol, se cuenta con las descripciones que Ian Hancock (1980a, 1980b, 1986, 1987) ha llevado a cabo en la comunidad texana de Brackettville. Hancock (1987, p. 270) nos ofrece un estudio comparativo en donde sugiere que la separación, aunque breve tanto temporal como física, ha causado solamente algunos procesos evolutivos importantes que distinguen al CBI que se habla en Nacimiento y Brackettville, Texas del CBI que se habla en las Carolinas.

Hoy en día, los mascogos se refieren a su comunidad como Colonia El Nacimiento para distinguirse de la comunidad vecina de los indígenas kikapú a cuya comunidad se refieren como Ranchería El Nacimiento o Nacimiento de los Indios. Por lo tanto, empleo el nombre que tradicionalmente se les ha dado a ambas comunidades: me referiré como Nacimiento de los Negros o Nacimiento al hablar de la comunidad mascoga y a la comunidad de los kikapúes como Nacimiento de los Indios. Según datos del Instituto Nacional de Estadística, Geografía e Informática (1996), en 1995 se calculaba que Nacimiento de los Negros tenía aproximadamente 247 habitantes mientras que Nacimiento de los Indios tenía 293.

Principalmente, me propongo en el siguiente trabajo trazar una historia lingüística para la comunidad en cuestión, basándome tanto en las referencias históricas disponibles como en la memoria colectiva y la evidencia histórica con que cuenta la comunidad misma. En el trabajo, concilio dos fuentes que nos proporcionarán datos y pruebas que nos 
permitirán delinear la trayectoria lingüística del grupo. Primero, utilizaré aquellos aspectos de la historia escrita que me son disponibles y que se centran en los principales acontecimientos que han experimentado los mascogos como grupo en el área fronteriza. Como tendencia general, estas fuentes históricas desestiman los aspectos lingüísticos que más me interesan aquí: el monolingüismo grupal, sobre todo las posibles diferencias entre géneros o grupos etarios; el bilingüismo, también relacionado a factores sociales; y las actitudes que los miembros del grupo y sus vecinos inmediatos podrían tener hacia la lengua o lenguas en contacto.

Segundo, incorporo al estudio el análisis de narrativas recogidas en entrevistas sociolingüísticas que se hicieron entre algunos informantes, la mayoría de ellos de edad ya muy avanzada, que habitan en la comunidad y que asumen una identidad mascoga por ser descendientes directos de padre o madre de origen afroseminol. Las narrativas forman parte de un pequeño corpus de entrevistas grabadas de naturaleza informal que se llevaron a cabo en Nacimiento de los Negros. En gran parte la conversación se centró en temas que consideramos de importancia para nuestro estudio: actitudes lingüísticas, identidad étnica y de grupo, historia de la comunidad, principales problemas que enfrenta la comunidad, relación con las comunidades vecinas e importancia de la comunidad en la región. Me apoyo en los testimonios orales que aparecen en la narrativas, dada la falta de referencias escritas que aluden directa o indirectamente a los patrones lingüísticos pasados de la comunidad. Me interesa asimismo trazar, a través del análisis narrativo e histórico, las implicaciones sociales y culturales que una situación de contacto de lenguas comprende para la comunidad minoritaria de los negros mascogos que se encuentra en una fase final del proceso de desplazamiento lingüístico y en plena integración hacia una cultura nacional dominante. Se describen las consecuencias que conlleva el desplazamiento lingüístico y se determinan algunas de las causas sociales e históricas de las cuáles probablemente se origina.

\section{Contacto cultural}

Son muchos los estudios que concuerdan en que el contacto lingüístico implica, en la mayoría de los casos, el contacto cultural 
(Weinreich, 1979, p. 5; Silva-Corvalán, 1989, p. 170). De hecho, para Silva-Corvalán (1989, p. 170), el estudio del contacto lingüístico debería de tomar en cuenta los aspectos sicológicos y socioculturales que entran en juego en el ámbito del contacto. Esto no sorprende puesto que la mayoría de los estudios coinciden en que la lengua es parte fundamental de lo que llamamos cultura. Hamer y Blanc (1989, p. 116), sugieren que como individuos nuestra conciencia cultural, es decir, el reconocernos como parte de una cultura -nuestra propia cultura- se da en la medida en que reconocemos la existencia de otras culturas dentro o fuera de nuestro entorno social. Desde este punto de vista, podríamos suponer que la diferencia entre dos grupos étnicos o culturales podría percibirse mucho más en la medida en que ambos grupos difieran en una mayor cantidad de rasgos culturales distintivos, tales como la lengua, raza, religión o estatus social. Indudablemente, en situaciones de contacto, la lengua sería un rasgo que se destacaría a la hora de formar una identidad cultural, o bien podría llegar a ser un marcador sociocultural con la capacidad de definir la adhesión de grupo.

Para Herzfeld (1994, p. 116), el tipo de contacto cultural y la manera en que éste se desarrolla tras todo encuentro depende en parte de las relaciones entre ambos grupos: de la imposición de un grupo y por lo tanto de la subordinación del otro o incluso de una posible relación de equilibrio. Si uno de los dos grupos ejerce el dominio político y económico sobre el otro, esto generalmente deriva en una relación de imposición; la relación inversa es la subordinación, en donde uno de los dos grupos se somete política, económica e incluso culturalmente. Herzfeld (1994, p. 116) propone que el contacto entre dos comunidades podría derivar en cualquiera de los siguientes escenarios: en un primer escenario, el grupo originario a la región de contacto podría sobreponerse, lo cual implicaría la subordinación del nuevo grupo; en un segundo, el grupo inmigrante se podría sobreponer, lo cual implicaría la subordinación del grupo original; y en un tercero, ningún grupo se sobrepondría. A la vez, los distintos escenarios socioculturales podrían derivar en distintos escenarios lingüísticos. Por ejemplo, el grupo que se sobrepone podría implantar su lengua u optar por utilizar la del grupo subordinado. Incluso, se le podría prohibir al grupo subordinado el uso de la lengua original o se podría limitar su uso a los dominios más íntimos. 
Lo que sí es seguro es que los factores socioculturales e históricos influyen sobre el resultado del contacto, uno de los cuales podría ser la aculturación del grupo minoritario. Berry (1980) sugiere que bajo una situación de aculturación, el comportamiento de un grupo se convierte más como el de otro. El mismo autor matiza el concepto de aculturación y propone los siguientes tipos: el proceso de asimilación ocurre cuando el grupo minoritario cede su identidad cultural y llega a diluirse en la sociedad mayoritaria; el proceso llega a su fin cuando los miembros del grupo en cuestión se sienten miembros del otro grupo y el otro grupo los acepta como miembros plenos. En el caso de la integración, el grupo llega a ser parte indivisible de la sociedad dominante, aunque se logra mantener diferencias culturales en diferentes niveles que lo distinguen del grupo dominante. La segregación se da cuando el grupo dominante reitera las diferencias culturales de ambos grupos e impide cualquier tipo de integración, en estos casos es común llegar a la marginalización social del grupo subordinado; mientras que la separación se presenta cuando el grupo subordinado decide sostener las diferencias, en estos casos, se podría optar por cierto grado de separación social.

La experiencia histórica de los mascogos ofrece la oportunidad de intentar reconstruir el pasado lingüístico del grupo, por lo que en lo que sigue hago un recuento del proceso de aculturación de los mascogos en El Nacimiento, tomo en cuenta algunos factores sociales e históricos que probablemente determinaron los proceso de aculturación en la comunidad. Al hacer el análisis de los datos orales, veo muy particularmente la manera en que la lengua, raza, religión o estatus social han destacado en la comunidad a la hora de formar una identidad cultural. Es muy posible que la lengua haya sido un rasgo que se destacaría a la hora de formar dicha identidad cultural, y que indudablemente debió haber sido en algún momento un marcador sociocultural con la capacidad de definir la adhesión al grupo.

\subsection{Difusión y pérdida en la aculturación}

La difusión o intercambio de rasgos culturales, incluyendo los lingüísticos, es un resultado lógico del contacto. Para Weinreich (1979, p. 5) la vecindad que se desarrolla entre dos grupos o más que comparten 
un espacio físico puede derivar en la difusión y en la eliminación de características culturales. Se podría suponer que casi cualquier grado de aculturación comprende una pérdida de rasgos distintivos, es decir, conforme se incorporan características ajenas al grupo podrían abandonarse algunas de las características únicas al grupo antes del contacto. Algunos estudios que se han dedicado a describir y explicar el contacto cultural, con particular interés en el aspecto lingüístico, han demostrado una tendencia unilateral del proceso (PeÑalosa, 1981, p. 171), a saber, que en muchos casos uno de los dos grupos generalmente se convierte en receptor y el otro en transmisor de una de las culturas en cuestión. Esto generalmente sucede a través de la adopción o imposición de la lengua o cultura que se percibe como dominante.

Anteriormente enumeré los distintos escenarios lingüísticos que podrían ocurrir tras el contacto cultural; con frecuencia, esas categorías se remiten a precedentes históricos que se caracterizaron por la expansión de potencias y grupos con fines colonialistas, imperialistas y mercantiles. Sin embargo, en el contexto histórico actual muchos de los casos de contacto, que presentan además procesos de aculturación, suceden dentro de la fuerza arrolladora de la nación-estado que busca la integración nacional, a través de la asimilación cultural y lingüística de los grupos minoritarios que se perciben como causa de retraso económico o posible amenaza política para la integridad territorial.

Todo caso de desplazamiento lingüístico implica el cambio en el uso habitual de una lengua por el uso habitual de otra (WEINREICH, 1979, p. 106) y las razones por las cuales un grupo acabaría por adoptar el uso cotidiano, casi exclusivo, de la lengua del grupo con que entra en contacto han sido motivo de una seria y larga discusión. Se ha propuesto que diversos factores sociales, externos a la lengua, parecen tener cierto efecto que precipita o posterga el proceso de desplazamiento cultural y lingüístico. La Figura 1 hace un recuento de algunos de los factores extralingüísticos que Romaine (1993, p. 41) y Grosjean (1982, p. 107) han enumerado como posible causa del mantenimiento o desplazamiento de las lenguas minoritarias. 


\begin{tabular}{|c|c|}
\hline RoMAine $(1993$, p. 41$)$ & GROSJEAN $(1982$, p. 107) \\
\hline - el tamaño del grupo & - el tamaño del grupo \\
\hline - la religión como factor de coherencia & - la religión como factor de coherencia \\
\hline $\begin{array}{l}\text { - la educación (ligada a la lengua de } \\
\text { enseñanza) }\end{array}$ & $\begin{array}{l}\text { - la educación (instrucción en la lengua } \\
\text { dominante o minoritaria) }\end{array}$ \\
\hline - el nivel socioeconómico & - el dinamismo socioeconómico \\
\hline - el patrón de asentamiento & $\begin{array}{l}\text { - la concentración del grupo dentro de } \\
\text { cierta área geográfica }\end{array}$ \\
\hline $\begin{array}{l}\text { - los lazos con el lugar de procedencia } \\
\text { en caso de inmigrantes }\end{array}$ & - el aislamiento geográfico \\
\hline $\begin{array}{l}\text { - el grado de casamientos con miembros } \\
\text { del grupo dominante }\end{array}$ & - los distintos periodos de nacionalismo \\
\hline \multirow{3}{*}{$\begin{array}{l}\text { - las actitudes de cada grupo y las } \\
\text { actitudes oficiales hacia las lenguas en } \\
\text { contacto }\end{array}$} & - las actitudes hacia su propia lengua \\
\hline & $\begin{array}{l}\text { - la manera en que el grupo ve su } \\
\text { condición migratoria (permanente o } \\
\text { temporal) }\end{array}$ \\
\hline & $\begin{array}{l}\text { - la fuerza asimilatoria de la sociedad } \\
\text { estadounidense. }\end{array}$ \\
\hline
\end{tabular}

Figura 1. Factores sociales que afectan el mantenimiento o desplazamiento lingüístico.

El patrón de integración hacia una cultura nacional dominante generalmente comprende una primera fase en la que una comunidad monolingüe adquiere cierto grado de bilingüismo. Sucesivamente este bilingüismo se acompaña de la pérdida de los contextos de uso de la lengua minoritaria hasta que la lengua dominante progresivamente la remplaza en todos los contextos. Peñalosa (1981, p. 175) sugiere que en todo caso de desplazamiento lingüístico hay una fase intermedia de bilingüismo mientras el grupo va de una fase monolingüe a otra y que este tipo de transición es el que generalmente ocurre entre grupos inmigrantes en los Estados Unidos a lo largo de tres generaciones. 


\subsection{El contacto español-CBI}

El impacto lingüístico del contacto a nivel social entre hablantes de lenguas distintas en una misma región, ha resultado en el surgimiento de lenguas pidgin que posteriormente experimentan un proceso de criollización. Le Page y Tabouret-Keller (1985, p. 17) conciben al pidgin como una lengua que surge principalmente para servir de lengua franca en situaciones de intercambio y cuya importancia resta en ser la lengua común entre estos hablantes de lenguas distintas; mientras que el criollo emerge en una situación en la que el pidgin ha cobrado más importancia que cualquier otra lengua en la comunidad y se ha convertido en la lengua de adquisición entre los nuevos miembros de la comunidad, es decir, los niños. El pidgin emerge ante una necesidad comunicativa entre individuos que no poseen una lengua en común y como característica principal el pidgin tiende a ser una variedad simplificada y reducida en comparación con la lengua lexificadora. Posteriormente, la etapa de creollización o expansión lingüística en la cual emerge un mayor número de categorías gramaticales en vías hacia un sistema lingüístico más complejo muy comúnmente es la etapa en la cual el pidgin pasa a ser la lengua nativa del grupo (Romaine, 1988, p. 41).

Para situar el caso de los mascogos dentro del entorno lingüístico de los CBI, empiezo por hacer un recuento de algunas de las situaciones en las que los CBI se encuentran en contacto con el español. Presto especial atención a la costa atlántica centroamericana, dada la preponderancia que ha mantenido ésta como lugar de contacto hasta hoy en día y porque una parte importante de la población, desde Belice hasta Panamá, es de origen negro y hablante de un CBI. Sin embargo, a pesar de que el uso de los CBI es común entre la población de esta área tan extensa, Belice es el único país centroamericano en donde el CBI predomina numéricamente; en los demás casos, los CBI se hablan en situaciones un tanto adversas por ser el español lengua administrativa y mayoritaria. Así que, con la excepción de Belice, el CBI tiende a ser la lengua minoritaria en la región.

Sin lugar a dudas, la larga presencia de los CBI en el área ha servido para contrarrestar la preponderancia del español. Por ejemplo, el CBI en la costa atlántica de Nicaragua se remonta a principios del siglo diecisiete: es el resultado de las incursiones inglesas en la 
región (HoLm, 1978, p. 1, 1983a, p. 95). El CBI en la costa atlántica de Honduras tiene una historia común con el CBI en Nicaragua y aparentemente data de la misma época. Holm (1983b, p. 95) nota que ambos CBI preceden a aquellos que se desarrollarían en Jamaica casi un cuarto de siglo después. Hubo además en casi toda la región y durante varias etapas históricas cierto refuerzo, aunque modesto, de hablantes de CBI. En algunos casos Gran Bretaña se ve forzada a ceder algunas de las islas más cercanas a las costas centroamericanas por lo que sus territorios y poblaciones llegan a formar partes de los distintos países centroamericanos; tal es el caso de las Islas de la Bahía, que en 1872 pasan a formar parte del territorio hondureño y cuyos pobladores son mayormente hablantes de inglés y de CBI (WARANTZ, 1983, p. 71-72). De manera similar, a principios del siglo veinte, las islas de Providencia y San Andrés, cuya población en su mayoría habla un CBI, pasan a formar parte del territorio colombiano (WASHABAUGH, 1983, p. 156157).

Hubo también hablantes de CBI que llegaron a la región por cuestiones laborales. En Costa Rica las primeras inmigraciones en masa de trabajadores antillanos, principalmente jamaicanos hablantes de un CBI, ocurren desde 1865, los trabajadores negros participan principalmente en la construcción ferroviaria (DunCAN Y MELÉNDEZ, 1981, p. 65). De manera similar, las primeras inmigraciones antillanas hacia Panamá se ligan al trabajo ferroviario que se lleva a cabo de 1850 a 1880 (HerzFeld, 1983, p. 150; Thomas-Brereton, 1993, p. 37-46). Fuera del istmo centroamericano, la primera inmigración en masa de negros hablantes de inglés y de CBI llega a la República Dominica en 1824 bajo el dominio haitiano de la isla cuando se concede a un grupo de libertos norteamericanos poblar parte de la Península de Samaná y otros puntos de la isla (Hoetink, 1984, p. 68-69). Cuba, Puerto Rico y otras partes de la República Dominicana también han recibido inmigración negra de regiones hablantes de CBI durante distintos periodos de su historia aunque en números más reducidos (LIPSKI, 2005, p. 40). A la lista, se pueden añadir los casos de Belice (HoLm, 1983a, p. 11) y Trinidad y Tobago (Moodie, 1982, p. 206) cuya lengua mayoritaria es un CBI, pero que mantienen minorías hispanohablantes dentro de sus territorio. 
Pero si bien el contacto entre los CBI y el español ocurre en todos los países antes citados, no sería exagerado el afirmar que nos encontramos ante un fenómeno poco documentado. Holm (1983a, p. 1) se refiere a estas variedades de inglés y CBI como el secreto mejor guardado de la América Central ("Central America's best kept secret"). En un estudio sobre el CBI en Centro América, Lipski (1986) analiza este tipo de contacto desde un punto de vista opuesto a lo que sucede en el caso del español en los Estados Unidos donde el inglés goza de prestigio oficial ante el español en todas las esferas de uso; por lo tanto, el español es la lengua minoritaria que ve sus usos restringidos al ámbito familiar principalmente. En un contexto caribeño, con la excepción de Belice y Trinidad y Tobago son con frecuencia los CBI los que se reservan para el ámbito familiar casi de manera exclusiva.

Holm (1983a, p. 11) sugiere que la diversidad que existe entre los CBI que se hablan a lo largo del litoral atlántico centroamericano refleja la realidad histórica de cada una de las comunidades que los hablan. Sería lógico pensar que en cualquier situación de contacto, la supervivencia o decadencia de una cultura minoritaria depende en buena medida del grado de contacto que se da con la lengua mayoritaria y dominante, por lo que cualquier análisis que intente definir las condiciones en las que se encuentra una lengua minoritaria en una etapa definida de su historia deberá tomar en cuenta los antecedentes históricos regionales o nacionales que ocurren a su alrededor. Por ejemplo, en Costa Rica Duncan y Meléndez (1981) dividen el impacto de la sociedad dominante sobre las culturas de origen africano y viceversa en tres fases: la fase del negro africano, bajo el sistema agrícola colonial, se caracteriza por la segregación racial de los grupos; la fase del negro antillano, bajo el sistema de plantación en empresas fruteras norteamericanas, se caracteriza por el aislamiento entre ambos grupos; y la fase del negro costarricense, bajo reformas legislativas y mayor predominio del gobierno central sobre la región de la costa atlántica, se caracteriza por la integración del negro a la cultura nacional en todos los ámbitos. De manera un tanto similar, Thomas-Brerenton (1993, p. 37-46) sugiere que en Panamá el impacto de la cultura dominante sobre la cultura afroantillana y viceversa se rige también por patrones socioeconómicos que se imponen durante las fases de la explotación bananera de la 
United Fruit Company, de la construcción del canal por compañías norteamericanas y finalmente de la integración del antillano a la vida nacional.

El contacto entre el español y el CBI en El Nacimiento se rige también por los eventos históricos bajo los cuales se da dicho contacto, por lo que la manera en que la cultura dominante mexicana percibe al nuevo grupo y en que el grupo se percibe a sí mismo es efecto directo de la función que éste último desempeña en la comunidad durante las distintas épocas históricas a través de las cuales atraviesa la comunidad lingüística. Hernández (2003) propone que dentro del contexto de la frontera, la vida cultural de los negros mascogos se rige y acopla de acuerdo a cuatro etapas sociohistóricas: 1) la etapa de arrendamiento militar y persecución, 2) la etapa de transición y neutralidad, 3) la etapa de fuerte integración a la cultura nacional y 4) la etapa de inmigración y dependencia económica. Por lo que a continuación analizo la manera en que algunos de los factores que se señalan en la sección anterior influyen en el mantenimiento y desplazamiento del CBI en la comunidad de los negros mascogos en cada una de las cuatro etapas históricas propuestas. Asimismo, determino los patrones lingüísticos y de identidad de grupo, pero agrego los testimonios orales que forman parte de las narrativas y que respaldan dichas divisiones sociohistóricas.

\section{DE FLORIDA A NACIMIENTO: FORJANDO UNA IDENTIDAD DE GRUPO}

La defensa ante los embates de los esclavistas ingleses y después norteamericanos ayudó a forjar una identidad de grupo entre los cimarrones negros que huyeron de las Carolinas y Georgia hacia la Florida. La identidad étnica y cultural de los afroseminoles, y de sus descendientes mascogos después de ellos, adquirió cohesión ante la urgencia por sobrevivir y mantener su libertad. Desde los siglos dieciséis y diecisiete, una buena parte de los cimarrones negros que huían de las colonias inglesas se dirigía a la Florida, donde las autoridades españolas otorgaban tierra a cambio de un servicio militar (Porter, 1996; Wright, 1986, p. 86). Este tipo de arrendamiento militar continuó hasta que los españoles cedieron la Península en 1819, año en que la mayoría de los colonos negros fueron trasladados a Cuba. 
Sin embargo, algunos afroseminoles permanecieron en la Península viviendo entre los indígenas de la región a quienes pagaban un tributo en grano a cambio de su libertad (PORTER, 1996, p. 4-5; Wright, 1986, p. 75-4). Durante los años en que los afroseminoles permanecieron en la Florida, se vieron involucrados en las diferentes guerras y escaramuzas entre España y los Estados Unidos; y en varias ocasiones formaron alianzas con los indígenas, para luchar contra el gobierno norteamericano (RoDRíGUEZ, 1995, p. 101). Esta relación perduró hasta 1842 cuando los Estados Unidos finalmente aseguró el control de la Península y los afroseminoles fueron trasladados al estado de Oklahoma (RodRíGuez, 1995, p. 101).

Es bajo este patrón de constante beligerancia y arrendamiento militar que los negros mascogos, junto con los indígenas seminoles y kikapúes, llegaron a México con la promesa de recibir un asentamiento a cambio de ayudar en la pacificación de la frontera de Coahuila (Mulroy, 1993, p. 55-56). Parte de este evento histórico se resume en el primer comentario que nos ofrece un informante de 72 años, que vivió mucho tiempo en Brackettville, pero que regresó a vivir a Nacimiento desde hace quince años. Aunque el informante habla español, se optó por entrevistarlo en inglés, dada la facilidad del entrevistador.

(1) HF: I know that they came over here running from slavery, so... that's one part of it, that it was better here then it was over there (NAC HF-H72) ${ }^{2}$

HF: Sé que vinieron aquí huyendo de la esclavitud, así que... eso es parte, que era mejor aquí que allá

El informante anterior comenta sobre la reubicación a Nacimiento que en su momento histórico debió haber significado una mejor situación para el grupo, aun cuando en las narrativas predominó una amnesia histórica en cuanto a las causas más específicas de la reubicación.

Como norma general, los informantes no comentaron sobre las causas que iban más allá del rechazo a la esclavitud, que obviamente motivó la huída de los Estados Unidos. Este mismo desconocimiento histórico se hace reiterativo en las demás entrevistas, como lo demuestra el siguiente comentario. 
(2) DG: Mi papá era de aquí, pero su, su mamá era de Estados Unidos, mi abuela...

Ent: ¿De qué parte?

DG: No, no sé... Mi mamá era de Estados Unidos también, sus padres también, pero cuando corrieron de, de por allá, fue cuando ellos vinieron

Ent: Nunca le contaron la historia de...

DG: No, no mi madre a mí, mi padre- nunca me contaron nada, pa' qué le voy a echar mentiras

Ent: ¿Le gustaría saber sobre la historia?

DG: Nomás me dijo que habían corrido de allá (NAC DG-M77)

Una constante entre los distintos informantes es el conocimiento que hay respecto al lugar en los Estados Unidos de donde salió el grupo inicial, ya que en algunos casos se sabe el lugar de nacimiento de algún antepasado, por lo tanto, la información se basa casi siempre en información de segunda mano.

(3) DL: ellos como dicen que venían de allá del otro lado, cuando pos ellos corrieron pa'acá, mis gentes que...

Ent: y le contaron de cómo pasó eso

DL: no pos, dicen que... mi 'buelito, él decía que venían de Florida y que unos 'bían nacido en Oklahuma (NAC DL-M64)

Hay entre los más jóvenes una aflicción por desconocer ese pasado que hasta hace poco los definía como grupo.

(4) DM: no, en particular no, pues es que como antes, como le digo yo, yo de niña no tenía ni siquiera idea de cómo, cómo había venido nuestra gente vedá los señores grandes los que ya no están con nosotros, no tenía ni siquiera una idea y después de casada pues con mis hijos eh... este ayudándolos este a que ellos este... pues cuando están en la secundaria pues viendo que ellos van a la escuela me empecé a informar o a interesar u poco más en lo que es este... eh, saber de cómo habíamos venido aquí de cómo vivía la gente antes y poco a poco veda le he venido dando valor vedá en realidad a lo que es la tribu y a lo que es este nuestra comunidad (NAC DM-M32) 
Los testimonios que hasta aquí hemos visto demuestran el poco conocimiento que se tiene en la comunidad misma sobre el pasado histórico común. En los próximos apartados se verá cada una de las etapas sociohistóricas que se proponen, pero añado los testimonios extraídos de las narrativas que aportan información que ratifica la propuesta anterior.

\subsection{Arrendamiento militar y persecución}

El arrendamiento militar que proveían los afroseminoles a las autoridades mexicanas fue en cierto modo la principal actividad que los llevó a México y sirvió desde muy temprano como factor distintivo entre éstos y sus vecinos ya en territorio mexicano. El asentarse en Nacimiento, ubicado a una distancia considerable de cualquier población mexicana importante, sirvió además de factor cohesivo que permitió hasta cierto punto la continuidad cultural y la transmisión lingüística. Si bien numéricamente los mascogos se vieron siempre superados por la población mestiza que los rodeaba, el aislamiento geográfico contribuyó a fortalecer la cohesión cultural y por lo tanto lingüística de la comunidad.

He argüido que en esta etapa histórica, son la ubicación espacial y la actividad principal del grupo lo que ayuda a mantener una identidad cultural distinta a sus vecinos más cercanos, pero al preguntarle a la siguiente informante sobre lo que ella sabía de esa etapa de arrendamiento, respondió:

(5) Ent: Pero de aquel entonces cuando se peleaban contra los indios, ¿hay una leyenda o un cuento de, de eso?

MV: pos de eso no, no le sabría decir porque no sé, no sé pues ya la mera verdad ya no me acuerdo

Ent: ¿Qué sus papás no le contaban mucho?

MV: No muncho asina, pos yo cuan- nomás crecí yo, me jui pal ejido Morelos allá vivía mi otra güelita. (NAC MV-M64)

Aunque es poco lo que nos dicen los testimonios orales sobre este periodo, el más lejano temporalmente, es de suponer que un resultado directo de la inestabilidad social y geográfica haya sido la creación de 
redes sociales estrechas de parentesco, amistad y vecindad, que en el caso de los mascogos favorecieron el mantenimiento lingüístico del CBI en la comunidad (c.f. MAC KAY, 1992). El aislamiento geográfico del grupo entre 1850 cuando los negros mascogos cruzaron la frontera y 1875 cuando la mayoría regresó de La Laguna y Brackettville a Nacimiento de los Negros debió haber frenado también su integración a la cultura mestiza de la región.

\subsection{Transición y neutralidad}

Durante la segunda mitad del siglo XIX los negros mascogos mantuvieron una estrategia de neutralidad en los asuntos políticos que se desarrollaban en México; mantuvieron la misma práctica a principios del siglo XX durante la Revolución Mexicana. Su condición como grupo inmigrante podría explicar dicha actitud; sin embargo, el individuo en la comunidad se identificaba ante todo como negro o negro mascogo antes que como mexicano o norteamericano en algunos casos.

Por otro lado, durante esta etapa se mantuvieron lazos estrechos con la comunidad de Brackettville entre 1870 y 1914 (Mulroy, 1993, p. 181). Hasta mediados de los años treinta los dos países permitían el paso libre entre las dos comunidades, situación que sin duda favorecía el uso del CBI como lengua franca. Tradicionalmente, se preferían los matrimonios entre mascogos o seminoles, como se autodefinía el grupo de Brackettville (Hancock, 1980a, p. 309; Mulroy, 1993, p. 181). El siguiente testimonio parece confirmar la movilidad que caracterizó a las dos comunidades fronterizas durante esta época.

(6) Ent: Where were you born?

HF: Here in Nacimiento.

Ent: But you haven't always lived here.

HF: No, no I lived 42 years in United States.

Ent: United States? And how were you able to do that?

HF: Oh, my grandmother, crossed- took me across (NAC HF-H72)

Ent: ¿Dónde nació?

HF: Aquí en Nacimiento. 
Ent: Pero Ud. No siempre ha vivido aquí.

HF: No, no viví 42 años en los Estado Unidos.

Ent: ¿Estados Unidos? ¿Y cómo pudo Ud. Hacer eso?

HF: Oh, mi abuela, me cruzó- me llevó para el otro lado.

Asimismo, se hace referencia a la experiencia de sus padres y abuelos que de la misma manera nos demuestra la movilidad que existió en un momento entre las dos comunidades y a los lazos que se mantenían entre Nacimiento y Brackettville.

(7) Ent: Where was your dad born?

HF: In Brackettville and-

Ent: And your mom?

HF: In Brackett, I don't know in what part of Brackett, but somewhere around there.

Ent: Why did they decide to come here?

HF: Well, they came here during, during the Depression, they moved over here, where my grandma and my grand pa lived, they got land and they moved over here to live.

(NAC HF-H72)

Ent: ¿Dónde nació su padre?

HF: En Brackettville y-

Ent: ¿Y su madre?

HF: En Brackettville, no sé en qué parte de Brackett, pero en algún lugar cercano.

Ent: ¿Por qué decidieron venir aquí?

HF: Bueno, vinieron durante, durante la Depresión, se mudaron para acá, donde my abuela y mi abuelo vivían, obtuvieron tierra y se mudaron para acá.

Dentro de esta narrativa, es obvia la ventaja que ofrecía el poder desplazarse de un lado a otro de la frontera en casos en los que uno de los dos países experimentara problemas socioeconómicos.

En 1880 cuando terminó la amenaza indígena en la frontera (Mulroy, 1993, p. 133) terminó también el patrón de arrendamiento militar que había caracterizado a los negros mascogos. Por primera vez los negros mascogos se dedicaron a la agricultura y ganadería, sin poder recurrir al arrendamiento militar como forma de vida. Empezó también 
la integración de la comunidad a la vida económica de la región con el arrendamiento de tierra a los campesinos mestizos (Mulroy, 1993, p. 172-173). Podría suponerse que en esta etapa la integración de los mascogos en una mayor medida a las faenas del campo constata también ya una mayor adaptación a la vida en tierras mexicanas. Este mismo informante se refiere al uso del inglés o CBI en Nacimiento durante la misma época.

(8) HF: I goes to Baptist Church.

Ent: And is the service in English or in Spanish?

HF: Spanish, nobody does the service in English anymore.

Ent: Before did they use to?

HF: Yeah, years back before the old timers left, when I was little they used to service- it was all English, back then people used to come from the United States.

Ent: People like?

HF: Ministers and the old timers from Brackett, you know, they lived over there and would come back here (NAC HF-H72)

HF: Voy a la iglesia Bautista

Ent: ¿Y la misa es en inglés o en español?

HF: Español, ya nadie da la misa en inglés.

Ent: ¿Antes solían hacerlo?

HF: Sí, en años anteriores antes de que se fueran todos los viejos, cuando era chico acostumbraban dar misa- era toda en inglés, antes venía gente de los Estados Unidos.

Ent: ¿Gente como quién?

HF: Ministros y los viejos de Brackett, usted sabe, vivían allá y venían para acá.

La presencia o ausencia de educación formal en español fue seguramente otro factor decisivo en la transmisión del CBI a las nuevas generaciones de mascogos principalmente en las primeras décadas del siglo XX. La falta de documentación del proceso en sí y de las actitudes en la comunidad hacia la creciente aculturación de la comunidad nos hace postular un posible escenario donde los hombres adquirían cierto grado de bilingüismo para el trato con las autoridades y para el comercio mientras que los niños con acceso a la educación escolar, sin importar 
el periodo de tiempo, muy probablemente adquirían el español a través del sistema educativo.

La estabilidad de la comunidad probablemente dio inició a la aculturación de los negros mascogos. El proceso debe haber comenzado con el bilingüismo; en este caso probablemente de los hombres, el grupo que participaba más en los tratos económicos con las comunidades vecinas de la región. Es probable también que la mujer en la comunidad haya tenido menos oportunidades de contacto con la cultura dominante; esto podría suponer dos cosas: primero, lingüísticamente la mujer se mantenía más conservadora; segundo, el CBI se conservaba como lengua del hogar en la fase inicial del desplazamiento lingüístico.

\subsection{Integración a la cultura nacional}

La integración de pequeñas culturas y lenguas minoritarias en muchos casos sigue patrones similares. Por ejemplo, Bradley (1992) acredita el desplazamiento lingüístico y la integración de la cultura ugon a la cultura tailandesa dominante a la accesibilidad de las comunidades después de la construcción de carreteras, al acceso de la educación en tailandés, a la llegada de tailandeses a las comunidades ugong y la gran cantidad de matrimonios con pobladores de comunidades vecinas. Noonan (1996) por su parte, atribuye el desplazamiento de la lengua chantyal en Nepal al contacto con la lengua nepalesa, a la actitud oficial hacia las lenguas minoritarias, a los matrimonios con nepaleses y las actitudes lingüísticas de los hablantes.

La integración cultural de los negros mascogos es muy parecida a los casos anteriores. Durante los años cuarentas, Nacimiento de los Negros formaba ya parte de la cultura nacional; contaba con una escuela primaria, una clínica comunitaria y dos iglesias: una bautista y otra católica (Mulroy, 1993, p. 175). El CBI en buena parte se mantenía vivo aunque a continuación nombro algunos factores que probablemente forjaron la fase final del desplazamiento. El hecho de que el CBI se mantuvo en la comunidad hasta hace una cuantas generaciones lo constatan los siguientes testimonios.

(9) Ent: Entonces, ¿antes hablaban mucho el inglés?

DG: Sí, el inglés tatoche y ya pos munchos ya se murieron. 
Ent: Entre sus hermanos, ¿se hablan Uds. mismos en inglés?

DG: Sí, en inglés, a veces venía gente de por fuera a buscar gente negra (NAC DG-M77)

Se ve aquí el desarrollo de una generación de bilingües, que claramente manejaban ya las dos lenguas.

(10) Ent: Y muchos de... de la gente de entonces hablaba inglés verdá

DL: todos lo hablaban más antes, lo poquito que nosotros sabemos, pos porque nos hablaba la gente verdá, más mi agüelito, mi mamá casi nunca nos habló

Ent: Pero su abuelito sí.

DL: Sí, pos mis abuelitos sí hablaban, ellos sí hablaban, sólo inglés porque hablaban poco mex-

Ent: ¿Su mamá por qué no lo aprendió?

DL: Sí lo sabía pero no nos hablaba, mira.

Ent: Entonces nomás lo hablaban entre ellos, pero no les...

DL: A nosotros no, mi güelita era la que siempre nos hablaba (NAC DL-M64)

Es interesante que la informante anterior alegue que en un momento tuvo un conocimiento pasivo del CBI, caso común en muchas situaciones de contacto en la que los menores no poseen una habilidad oral, pero pueden entender la lengua comunitaria. Por lo tanto, se comunican con los mayores, por ejemplo con los abuelos, en la lengua dominante, y los mayores les contestan en la lengua minoritaria.

En esta misma etapa, se desarrolló junto a Nacimiento de los Negros el Ejido Morelos un asentamiento de población mestiza dedicado a la agricultura. Los matrimonios entre negros mascogos y mestizos de las dos comunidades aumentaron considerablemente; algunos negros mascogos también se casaron con mestizos de otras poblaciones vecinas y aun de otras partes de México. Una de la informantes de mayor edad nos comenta lo siguiente sobre los casos de matrimonios mixtos.

(11) Ent: ¿Cuándo fue que la gente de Nacimiento se empezó a casar con gente de fuera de Nacimiento?

DG: Pos yo creo que desde que vinieron creo yo, pos cuando yo nací ya había gente mexicana casada con gente de color (NAC DG-M77) 
Otra informante nos confirmó esta tendencia al informarnos sobre el caso de su familia.

(12) DL: Mi papá era mexicano, como les digo, y mi mamá era negrita.

Ent: ¿Su papá de dónde era?

DL: De Músquiz, era de Músquiz y se casó con mi mamá, todas mis gentes por parte de ella eran negras (NAC DL-M64)

El ejemplo a continuación resulta interesante porque la informante ata la pérdida de la lengua original al alto grado de mestizaje que es evidente en la comunidad, y sugiere además que la exogamia ha tenido serias repercusiones en las habilidades lingüísticas de las generaciones más jóvenes.

(13) Ent: ¿Aquí en nacimiento queda ya poca gente negra?

DG: Casi no hay oiga, no hay... negros, negras como yo nomás mis hermanos y mis sobrinos, muchos son como la familia de mis sobrinos [mestizos], pero gentes grandes no hay que hablen en inglés, habrá una o dos, como Enrique, este Chito que los llevó, el papá de Chito es de este color [negro], aunque la mamá es mexicana, pelo liso como este muchacho (NAC DG-M77)

Es muy probable que en los hogares con matrimonios mixtos haya predominado el uso del español, sin importar el sexo del padre. Como resultado, el CBI no se transmitió a los hijos de matrimonios mixtos lo cual creó una generación esencialmente monolingüe, como podemos ver a continuación.

(14) Ent: Y... su mamá era de Chihuahua y su papá era mascogo y... ¿qué hablaban en la casa ellos?

MV: no pos siempre en español, en español, nomás mi papá sí nos quería hablar así en veces y nosotros nunca le pusimos atención a hablar el inglés, pues muy poquito entendemos, muy poquito, yo la mera verdá ya después 'taba yo arrepentida porque... no agarré de cómo nos decían los papás de hablar en inglés porque es muy bonito, a mí sí me gusta hablar en inglés pero pos no sé, yo nada más muy poquito lo que sé hablar. (NAC MV-M64) 
Aquellos hogares donde los dos padres eran negros mascogos seguramente mantuvieron el uso del CBI por lo menos en este dominio, aunque los hijos adquirieron el español a través del sistema educativo, situación que nos pinta la siguiente informante.

(15) Ent: ¿En aquel entonces hablaban español o hablaban lo que usted llamó tatoche?

DG: Pos este los que eran mexicanos pos hablaban mexicano y los que eran negros pos hablaban las dos idiomas

Ent: ¿Sus papás hablaban inglés o los dos?

DG: En la casa las dos hablaban porque los dos eran... mi mamá era negra, negra así como yo, mi papá no era eh... más light, pero era negro. (NAC DG-M77)

Indudablemente, este periodo favoreció el cambio de código en la comunidad como sería de esperarse en este ambiente de bilingüismo intenso. Hay en los testimonios solamente una alusión directa al bilingüismo intenso que debió haber caracterizado el habla de la comunidad. Incluso, aquí la informante nos da un ejemplo claro del cambio de código y nos ofrece también una evaluación personal de dicha modalidad: negativa, como es el caso en algunas comunidades bilingües.

(16) MV: pos yo más antes digo como oía a muchas personas que hablaban la mitá en, en inglés y la mitá en español pos yo me imaginé que no era lo correcto, que no era lo correcto lo que estaban hablando porque hay unas que dicían: gone to da house y luego le dicían algo en español pos no yo... se me imaginaba que no era lo correcto vedá. (NAC MV-M64)

Lo más probable es que el desplazamiento lingüístico se haya acelerado conforme disminuyeron los contextos de uso del CBI en la comunidad --según patrones variacionales de estilo. Los contextos más íntimos debieron favorecer su uso aunque la presencia de los hablantes mestizos monolingües en la comunidad seguramente restringió aún más los contextos. Por ejemplo, el español se utilizó exclusivamente en situaciones formales dentro y fuera de la comunidad, para tratar asuntos oficiales; en situaciones de educación formal también se prefirió el español puesto que hasta hoy los profesores vienen de fuera a impartir 
clases en la comunidad. Al contrario, se prefirió el uso del CBI en el contexto religioso. La iglesia bautista sirvió por algún tiempo como factor de cohesión. Sin embargo, al llegar los mestizos a la comunidad también llegó el catolicismo (de allí la tradición de mantener dos iglesias en la comunidad). Mientras que la primera favoreció el uso del CBI, la segunda se valió del uso del español porque los sacerdotes hasta hoy también vienen de fuera.

En torno al uso de la lengua hereditaria en la comunidad, se encontraron los siguientes comentarios.

(17) Ent: Did any of your parents speak Seminole?

HF: Yeah, everybody spoke Seminole back then.

Ent: Do you remember any of the Seminole?

HF: Well, not too many, cause it was a lot older people that- they were mainly gone when I was old enough to know any (NAC HFH72)

Ent:¿Alguno de sus padres hablaba seminol?

Hf: Sí, antes todos hablaban seminol.

Ent: ¿Recuerda algo de seminol?

HF: Bueno, no mucho, porque era la gente mucho más vieja quecasi todos ya se habían ido cuando yo estaba lo suficientemente grande para prender.

La mayoría de los entrevistados concuerdan en que la falta de una transmisión oral de las tradiciones de la comunidad, y quizás indirectamente de una transmisión lingüística, se debe a la costumbre misma de no incluir a los niños en las actividades y conversaciones de los adultos.

(18) Ent: Did your parents tell you any stories?

HF: No, old timers they didn't believe in us being around when they was talking.

Ent: Is it the same now?

HF: No (NAC HF-H72)

Ent:¿Sus padres le contaron alguna historia?

HF: No, los viejos no creían en que nosotros estuviéramos presentes cuando ellos hablaban entre sí. 
Ent: ¿Todavía es igual?

HF: No.

Otra narrativa que habla sobre los efectos de la falta de la transmisión de la lengua comunitaria a las nuevas generaciones la expone una de las informantes más jóvenes, quien expresa su añoranza por la pérdida del CBI y por la falta de convivencia con los ancianos que hablaban la lengua hereditaria.

(19) Ent: Según a lo que Ud. tiene entendido vedá, ¿cómo es que llegaron los mascogos aquí?

DM: Pues este... bueno mi abuelito él platicaba este que... pues en realidad pues no nos dijo mucho vedá nada más que o sea acerca de sus comidas de lo tradicional verdá de cómo hacían las gentes antes y-

Ent: ¿Como qué tipo de cosas le dijo?

DM: Pues él hablaba inglés, verdá, él cantaba muy bonito pero yo pienso que lo que faltó fue que él nos diera un poco más de su tiempo para que nosotros aprendiéramos a hablar el inglés, pero pues estábamos este... vivíamos aquí mismo, pero él estaba siempre ocupado en sus labores, él tenía muy poco tiempo así que fue muy poco lo que aprendimos de él. (NAC DM-M32)

El siguiente testimonio, atribuye la falta de transmisión al papel que jugo la madre, que no era mascoga, en su crianza.

(20) Ent: ¿Hubo una época en que usted entendió aunque sea tantito?

MV: pos cuando estaba más joven pos ahí andaba, me juntaba con la demás compañeras que sí- me gustaba mucho yo como hablaban ellos vedá, decía yo por qué no- pos mi papá- si mi mamá había sido mexi- este mascoga, a lo mejor nosotros habíamos aprendido algo como aprendían los demás pero como mi mamá es mexicana, no es igual porque el papá sale al campo y la mamá no, está de diario con sus hijos, por ejemplo si yo 'biera hablado inglés mis hijas lo hubieran hablado también (NAC MV-M64)

Es interesante ver que a pesar de que la informante anterior no tiene ningún tipo de instrucción formal para determinar los factores 
sociales que favorecen la transmisión y uso de una lengua minoritaria en una situación de contacto, el análisis de la situación familiar la llevan a proponer diferentes escenarios basados en el origen étnico de la madre.

Durante esta etapa ocurrió también la integración definitiva de la comunidad a las redes nacionales de comunicación: la construcción de carreteras unió a Nacimiento de los Negros con Músquiz, la población mestiza más importante de la región. Se transmitieron también las primeras emisoras de radio y con el tiempo de televisión en español. Al mismo tiempo, la comunicación con la comunidad de Brackettville no se interrumpió completamente, pero disminuyó considerablemente.

En el contexto nacional, esta etapa debió haberse marcado por una fuerte tendencia nacionalista. Una consecuencia directa fue el incremento en el subsidio al campo. Como resultado, en 1938 Lázaro Cárdenas ratificó el derecho de los negros mascogos sobre la región de Nacimiento de los Negros y concedió el derecho a terrenos adicionales anexos a la comunidad (PorTER, 1996, p. 225). Por otro lado, el nacionalismo se definía por una dicotomía que exaltaba el sentimiento antiextranjero (sobretodo norteamericano) y el sentimiento promexicano (GARCÍA MARSH, 1982, p. 166). Las palabras de la siguente informante resumen ese sentimiento promexicano que sienten aún todos los informantes mascogos que entrevistamos.

(21) DL: no sí los recuerdo, él jue jefe le digo, to'avía cuando 'taba Lázaro Cárdenas to'avía él era jefe de aquí de la Colonia, él jue muy mentado, Counte, le decían Counte, pero eran gentes que habían venido del otro lado. Le di- y... como le cuento de Juan Caballo, pero era por parte de mi esposo, ese John Horse que le dicen, era de parte de mi esposo, pero sus gentes también eran de allá y sus papás también, pero nosotros semos de México, aquí nacimos, semos criados aquí vedá, todo el derecho lo tenemos porque semos mexicanos (NAC DL-M64)

\subsection{Inmigración y dependencia económica}

En la fase final del desplazamiento, la pérdida de población a través de la emigración y la reubicación han acelerado la integración de 
la comunidad a la cultura nacional. La emigración ha seguido patrones tradicionales y responde a razones económicas: han emigrado hombres y mujeres jóvenes hacia ciudades en ambos lados de la frontera o en el interior de los Estados Unidos donde pueden ofrecer su mano de obra barata. La informante de mayor edad nos confirma este patrón.

(22) Ent: ¿Nacimiento siempre ha sido así?

DG: Sí, asina como lo ve, no pero pos muchos salieron de aquí... y otros pos se murieron (NAC DG-M77)

El contraste entre lo que se percibía como una comunidad más saludable demográficamente y la situación actual, marcada por la expulsión de la población más joven, se ve en el siguiente testimonio.

(23) Ent: Recuerda algo de Nacimiento cuando era niña

DL: Oh, pos era muy bonito, pero no vuelven los tiempos.

Ent: ¿Parecido a hoy?

DL: No, nunca porque antes había muncha gente, muy bonito, muy grande y había... 'taba este ejido, munchas gentes, no güelven los tiempos de antes, hoy la gente se está acabando, ya todos- puro retoño 'tan quedando (NAC DL-M64)

Por otro lado, los matrimonios entre negros mascogos y mestizos han intensificado la diáspora a las comunidades vecinas, sobre todo a Músquiz y al Ejido Morelos. La salida de la comunidad generalmente ha implicado el desconectarse aun más de la herencia cultural. La breve narración a continuación nos muestra que el proceso aculturación y mestizaje continúa en Nacimiento.

(24) Ent: ¿Entonces era muy común que se casaran en su época negros con mexicanos?

DL: Sí, mexicanos con los negritos, digo y había muchos mexicanos, pos casi todos están casados con mexicanos porque yo tengo mis hijas y todas 'tan casadas con mexicanos, nomás una hija mía 'ta casada con uno de su raza (NAC DL-M64)

Para los hombres, las oportunidades de trabajo en la comunidad se han limitado a las tareas del campo mientras que para la mujer han 
sido casi nulas. Esa falta de trabajo, como motivo de expulsión, se expresa en el siguiente testimonio.

(25) NV: Yo en lo personal me gustaría que hubiera fuentes de trabajo vedá para que la misma gente tuviera pues este pues tuvieran vedá trabajo y... y pues que... para que- o sea para ayudar a sus mismos hijos vedá para salir adelante porque como no tenemos fuentes de trabajo pues es difícil entonces yo creo que con que la gente se ocupara en algo pues estaría muy bueno porque así mismo, o sea cómo le podría decir, quisiéramos que la misma gente valorara lo que tenemos aquí (NAC NV-M33)

Por otro lado, lo incosteable que resulta para algunos el trabajar en el campo se expresa a continuación.

(26) DM: en gran parte la gente son agricultores y... la ganadería, pero no lo hacemos para beneficio sino que lo hace nada más para consumo de aquí mismo vedá porque es incosteable ya hacer- tenerlo para comerciarlo vedá porque pues no hay precios vedá, pero ya con el hecho de que lo téngamos para consumo de uno mismo ya es bastante (NAC DM-M32)

En años recientes, la situación migratoria de los kikapúes en Nacimiento de los Indios hacia los Estados Unidos ha creado una dependencia estrecha entre algunas familias de negros mascogos y kikapúes. La mayoría de los kikapúes viven parte del año en los Estados Unidos y parte del año en Nacimiento de los Indios. Con las divisas que obtienen, los kikapúes contratan a los negros mascogos para cuidar sus viviendas durante el tiempo que están en los Estados Unidos, para trabajar en las tareas del campo, para curtir cuero que utilizan en la elaboración de artesanías, para la construcción de sus viviendas tradicionales, para deshierbar terrenos y para otros trabajos temporales, como se constata a continuación.

(27) MV: Sí, pos 'horita... 'horita el trabajo de aquí de Nacimiento somos- con los inditos, se van aquí a coser tule, a, a curtir la tegua, el cuero y ya saben munchas mujeres de aquí, saben ya curtir el cuero y ahorita andan en la limpia, les hacen las casas les ayudan a hacer las casas y les pagan, como los inditos horita pos train dólares y aquí 
pues no hay ni uno- fuente de trabajo ni nada, eso es lo que queremos que el gobierno nos oiga, nosotros tanto pedir que nos pongan buena fuente de trabajo, eso es lo que queremos... (NAC MV-M64)

El estrecho contacto con los kikapúes ha tenido dos consecuencias principales: primero, en algunos miembros de la comunidad ha despertado el deseo de poder obtener la doble ciudadanía y con ello una mejor calidad de vida; mientras que en otros ha hecho obvio el grado en que los kikapúes han mantenido sus costumbres, tradiciones y lenguaje y la medida en que se han perdido en su comunidad.

Por otra parte, la inmigración hacia los Estados Unidos ha despertado un interés entre algunos negros mascogos por aprender el inglés, ya que los emigrantes generalmente regresan del norte con destrezas básicas o avanzadas. Algunos miembros de la comunidad sienten la pérdida del CBI y quisieran revertir el proceso de desplazamiento porque lo asocian con el inglés norteamericano. En este caso las nuevas generaciones ven el aprendizaje del CBI, equivocadamente por la asociación con el inglés, como benéfico y fuente de remuneración económica.

Indudablemente, El proceso de desplazamiento casi completo del CBI responde a la utilidad de uso que cada lengua ofrece a los hablantes en los distintos contextos sociales, por ejemplo en situaciones oficiales o laborales, y al prestigio social o económico de cada una. Actualmente el CBI lo habla solo un puñado de ancianos de edad avanzada y seguramente desaparecerá con esta generación. Los hablantes no tienen la oportunidad de utilizarlo entre sí porque viven en hogares diferentes. Además, el entrar en contacto no implica necesariamente el uso del CBI porque aun los últimos hablantes son bilingües; el uso de una u otra lengua responde al contexto de habla: un encuentro generalmente se daría en situaciones públicas y no íntimas.

\section{CONCLUSIÓN}

El desplazamiento lingüístico como resultado del contacto cultural en Nacimiento de los Negros inició la integración de los negros mascogos a la cultura nacional. Sin embargo, el contexto sociohistórico 
marcó la pauta del proceso de aculturación. En el presente trabajo ofrezco datos testimoniales que apoyan las cuatro fases sociohistóricas, que arguyo, influyeron en dicho proceso: 1) la etapa de arrendamiento militar y persecución, 2) la etapa de transición y neutralidad, 3) la etapa de fuerte integración a la cultura nacional y 4) la etapa de integración y dependencia económica. Se distinguen entre sí por el mantenimiento del $\mathrm{CBI}$ en la primera fase, el inicio de la desplazamiento en las siguientes y el desplazamiento casi total en la última. Actualmente, la identidad étnica de los negros mascogos se marca a través de funciones culturales comunitarias en su mayoría ritualizadas que resaltan la diferencia de la comunidad en el contorno regional. El desplazamiento del CBI requiere que las nuevas generaciones revaloren los actos culturales que han perdurado a pesar de la aculturación para suplantar la importancia que representaba la diferencia lingüística.

Es importante recalcar que el caso de los negros mascogos tiene repercusiones en el caso de los CBI que se hablan en Centro América en situaciones de contacto con el español. Los estudios que se han mencionado anteriormente describen una situación de desplazamiento con actitudes e implicaciones similares al caso de los negros mascogos. Sin embargo, la situación en Nacimiento de los Negros se ha acelerado debido a la ventaja numérica de la población mestiza en la región.

RECONSTRUCTION OF A LINGUISTIC PAST THROUGH PRESENT NARRATIVE: LANGUAGE, HISTORY AND IDENTITY IN THE El NACIMIENTO DE LOS NEgRoS

\section{AbSTRACT}

Nacimiento de los Negros, main settlement of the Black Mascogos, is located within the boundaries of the municipality of Músquiz, Coahuila, Mexico. Afroseminol Creole, a language closely related to the Creole variety spoken in the southern United States, was the community's original and majority language. In the following study, I trace the community's sociolinguistic history, based on the available historical references in the collective memory and in the historical evidence accessible to the community itself. I bring together two sources that will provide data and proof that will allow us to delineate the group's linguistic course. I draw from any available aspect of written history that focus on the main events experienced by the Mascogo as a group in the border region. I also incorporate the analysis of narratives 
gathered in sociolinguistic interviews that were made among older informants in the community who assume a Mascogo identity because they are direct descendants of an Afroseminol father or mother. The analysis points out that language shift, as a result of the cultural contact in Nacimiento de los Negros, began the integration of the Black Mascogos to the national culture. However, it was the sociohistorical context that set the pace of the acculturation process: 1) the period of military hiring and persecution, 2) the period of transition and neutrality, 3) the period of strong integration to the national culture, and 4) the period of integration and economic dependence. Periods are distinguished by the maintenance of Afroseminol Creole in the first phase, the beginning of language shift in the following, and the quasi-total shift in the latter.

KEY WORDS: identity, black mascogos, mexican linguistic mosaic.

\section{NOTAS}

1 Utilizo el término negro mascogo o mascogo en la discusión por ser éste el que se prefiere en la comunidad a la hora de marcar la diferencia entre alguien de origen afroseminol y alguien de origen mestizo sin aparente conexión racial o cultural con la comunidad: es decir, sin aparente herencia negra.

2 La información entre paréntesis es la siguiente: (Nac siglas del hablante / sexo: $\mathrm{H}=$ hombre, $\mathrm{M}=$ mujer / edad).

\section{BiBLIOGRAFÍA}

Berry, J. D. Acculturation as Varieties of Adaptation. En Padilla, A. (Ed.). Acculturation: Theory and Models. Washington: AAAS, 1980.

Bradley, David. The Disappearance of the Ugong in Thailand. En Dorian, Nancy C. (Ed.). Investigating Obsolescence: Studies in Language Contraction and Death. New York: Cambridge University, 1992.

CAsidy, Frederic G. Some Similarities Between Gullah and Caribbean Creoles. En Montgomery, Michael B. y Bailey, Guy (Eds.). Language Variety in the South: Perspectives in Black and White. Alabama: University of Alabama, 1986.

Duncan, Quince y Meléndez, Carlos. El negro en Costa Rica. San José, Costa Rica: Editorial Costa Rica, 1981. 
García, Marsh; Alma, María. Ideology and Power: A Study of the State Under Porfirio Díaz (1876-1911) and Lázaro Cárdenas (1934-1940). Ph.D. Thesis, Harvard: Harvard University, 1982.

Grosjean, François. Life with Two Languages: An Introduction to Bilingualism. Cambridge, MA: Harvard University, 1982.

Hamer, Josiane F. y Michel, H. A. Blanc. Bilinguality and Bilingualism. Cambridge: Cambridge University Press, 1989.

Hancock, Ian. Texan Gullah: The Creole English of the Brackettville AfroSeminoles. En Dillard, J. L. (Ed.). Perspectives on American English. The Hague: Mouton, 1980a.

Hancock, Ian. The Texas Seminole and Their Language. Austin, Texas: The University of Texas, $1980 \mathrm{~b}$.

Hancock, Ian. On the Classification of Afro-Seminole Creole. En Montgomery, Michael B. y BAILEY, Guy (Eds.). Language Variety in the South: Perspectives in Black and White. Alabama: University of Alabama, 1986.

HANCOCK, Ian. A preliminary Classification of the Anglophone Atlantic Creoles with Syntactic Data from Thirty-three Representative Dialects. En GILBERT, Glenn G. (Ed.). Pidgin and Creole Languages: Essays in Memory of John E. Reinecke. Honolulu: University of Hawaii, 1987.

Hernández, José Esteban. Aculturación e identidad en El Nacimiento de los Negros. Río Bravo: A Journal of Borderlands, n. 2, v. 1, p. 85-97, 2003.

Herzfeld, Anita. The Creoles of Costa Rica and Panama. En Holm, John (Ed.). Central American English. Heidelberg: Gross, 1983.

Herzfeld, Anita. Language and Identity: The Black Minority of Costa Rica. Revista de Filología y Lingüistica de la Universidad de Costa Rica, n. 20, v. 1, p. 113-142, 1994.

Hoetink, H. Los americanos de Samaná. En Ortiz, Dagoberto Tejeda (Ed.). Cultura y folklore de Samaná. Santo Domingo, República Dominicana: Alfa \& Omega, 1984.

Holm, John. The Creole English of Nicaragua's Miskito Coast: It's Sociolinguistic History and a Comparative Study of its Lexicon and Syntax. $\mathrm{Ph} . \mathrm{D}$. Thesis. London: University of London, University College, 1978.

Holm, John. Central American English: An Introduction. En HoLm, John (Ed.). Central American English. Heidelberg: Gross, 1983a.

Holm, John. Nicaragua's Miskito Coast Creole English. En Holm, John (Ed.). Central American English. Heidelberg: Gross, 1983b. 
Instituto Nacional de Estadística, Geografía e Informática. 1996. Coahuila. Conteo de Población y vivienda 1995. Resultados Definitivos. Tabulados básicos del XI Censo General de Población y Vivienda, 1990.

JONES-JACKSON, Patricia. Gullah: On the Question of Afro-American Language. Antropological Linguistics, n. 20, v. 1, p. 422-429, 1978.

Jones-JACKSON, Patricia. On Decreolization and Language Death in Gullah. Language in Society, 13, n. 2, p. 351-362, 1984.

Jones-JACKSON, Patricia. On the Status of Gullah on the Sea Islands. En Montgomery, Michael B. y Bailey, Guy (Eds.). Language Variety in the South: Perspectives in Black and White. Alabama: University of Alabama, 1986.

Jones-Jackson, Patricia. When Roots Die: Endangered Traditions on the Sea Islands. Athens, Georgia: University of Georgia, 1987.

Le Page, R. B. Y Tabouret-Keller, Andrée. Acts of Identity: Creole-Based Approaches to Language and Ethnicity. New York: Cambridge University Press, 1985.

LIPSKI, John. English-Spanish contact in the United States and Central America: Sociolinguistic Mirror images?. En Gorlach, Manfred y Holm, John A. (Eds.). Focus on the Caribbean. Philadelphia: John Benjamins, 1986.

LIPSKI, John. El español en el mundo: Frutos del ultimo siglo de contactos lingüísticos. En López: Luis A. Ortiz y LACORTE, Manel (Eds.). Contactos y contextos lingüísticos: El español en los Estados Unidos y en contacto con otras lenguas. Madrid: Iberoamericana, 2005.

MACKAY, C. J. Language Maintenance in Chipilo. International Journal of the Sociology of Language 96, 1992. p. 129-145.

Moodie, Silvia Maria. Trinidad Spanish Pronouns: A Case of Language Death in the Caribbean. En NASH, Rose (Ed.). Readings in Spanish-English Contrastive Linguistics, v. III. San Juan, Puerto Rico: Inter American University, 1982.

Mulroy, Kevin. Freedom in the Border: The Seminole Maroons in Florida, the Indian Territory, Coahuila, and Texas. Lubbock, Texas: Texas Tech University, 1993.

Noonan, Michael. The Fall and Rise of the Chantyal Language. Southwest Journal of Linguistics 15, n. 1-2, p. 121-135, 1996.

Peñalosa, Fernando. Introduction to the Sociology of Language. Rowley, MA: Newbury House, 1981.

Porter, Kenneth W. The Black Seminoles: History of a Freedom-Seeking People. Gainesville, Florida: University of Florida, 1996. 
RodRíguez, Martha. Historias de resistencia y exterminio: Los indios de Coahuila durante el siglo XIX. México, D.F.: Centro de Investigaciones y Estudios Superiores en Antropología Social, 1995.

Romaine, Suzanne. Pidgin and creole languages. New York: Longman, 1988.

Romaine, Suzanne. Bilingualism. Cambridge, MA: Blackwell, 1993.

Silva-Corvalán, Carmen. Sociolingüistica. Madrid: Alhambra, 1989.

Thomason-Brereton, Leticia C. An Exploration of Panamanian Creole English: Some Syntactic, Lexical and Sociolinguistic Features. Ph.D. Thesis. New York University, 1993.

Warantz, Elissa. The Bay Islands English of Honduras. En Holm, John (Ed.). Central American English. Heidelberg: Gross, 1983.

Washabaugh, William. The Off-shore Island Creoles: Providencia, San Andrés and the Caymans. En Holm, John (Ed.). Central American English. Heidelberg: Gross, 1983.

WeinReIch, Uriel. Languages in Contact. The Hague: Mouton, 1979.

Wright, J. Leitch. Creeks and Seminoles: The Destruction and Regeneration of the Muscogulge People. Lincoln, Nebraska: University of Nebraska, 1986. 